\title{
In Vivo Biomechanics of the Fingerpad Skin Under Local Tangential Traction
}

\author{
Qi Wang and Vincent Hayward* \\ Haptics Laboratory, Centre for Intelligent Machines, McGill University, 3480 \\ University Street, Montréal, H3A 2A7, Canada
}

\begin{abstract}
Small patches of fingerpad glabrous skin in human subjects were tested in vivo for their biomechanical properties under tangential loading and for large deformations. These conditions included stretching and shearing the skin at a length scale of $0.3 \mathrm{~mm}$ using an apparatus comprising a pair of piezoelectric benders arranged to increase the stiffness/free deflection tradeoff when compared to ordinary cantilevered benders. It was then possible to test the skin with up to $80 \%$ of tangential strain. With feedback control, it was also possible to create isotonic and isometric testing conditions. The results showed much variability across subjects and it was seen that the glabrous skin exhibited nonlinear stiffening in tangential traction. The skin was consistently more elastic across the ridges than along the ridges regardless of the location of the sample on the fingerpad. The skin behaved visco-elastically but relaxed about twice as fast than it crept. Finally, it was found that under large deformation, there was consistently an $80 \%$ of hysteretic loss for a wide range of loading conditions.
\end{abstract}

Key words: Fingerpad skin properties; In vivo tissue measurement; Biomechanics; Fingers.

Word count (main text): 3500 .

* Corresponding author. Centre for Intelligent Machines, McGill University, 3480 University Street, Montréal, H3A 2A7, Canada. Tel. +1-514-398-5006, Fax. +1-514-398-7348.

Email address: hayward@cim.mcgill.ca (Vincent Hayward). 


\section{Introduction}

An important function of the glabrous skin is to mediate tactile sensations. When the skin comes in contact with an object, mechanical loading causes it to deform from a relaxed state. Several types of mechanoreceptors signal these deformations to the central nervous system (CNS), ultimately resulting in the perception of the object's attributes such as shape or compliance. Many studies have attempted to identify the responses of these cutaneous receptors under various loads (Johansson and Vallbo, 1979b; Phillips and Johnson, 1981a; Johansson and Vallbo, 1983; Johansson and Westling, 1984, 1987; Srinivasan and LaMotte, 1987; Johnson and Hsiao, 1992; Edin and Johansson, 1995; Goodwin et al., 1997; Bisley et al., 2000; Johnson, 2001; Johansson and Birznieks, 2004). It would seem that a characterization of the biomechanics of the glabrous skin would contribute to the understanding of the tactile function since receptors respond to patterns of skin deformation (Goodwin and Wheat, 2004). Birznieks et al. (2001) suggested that different strain patterns caused by anisotropic material properties might account for the directionality of tactile afferent responses. Moy et al. (2000a) found that viscoelasticity had significant effect on tactile perception. Biggs and Srinivasan (2002) reported that some individuals were more sensitive to tangential forces than to normal forces on their forearms, but the opposite was true on their fingerpads. Taken together, these observations suggest that the biomechanics of the skin could have functional consequences for how mechanoreceptors in the skin are stimulated and for the type of signals sent to the brain.

Cauna (1954) argued with the aid of an analog model that the structure of papillary ridges acted

as "a magnifying lever mechanism for transmission of touch stimuli to the underlying receptors". Since then, there has been much interest in numerical models to elucidate the function of receptors embedded in the skin (Phillips and Johnson, 1981b; Srinivasan and Dandekar, 1996; Maeno et al., 1998; Moy et al., 2000a; Wu et al., 2003b; Gerling and Thomas, 2005). Lack of knowledge of the glabrous skin's properties led some authors to use numbers measured for the hairy skin. Others solved an inverse problem by matching the predicted shape with measurements, but the results were unreliable because of the assumptions required. Knowledge of the detailed behavior of the skin could also be of interest to tactile display designers (Moy et al., 2000b; Drewing et al., 2005; Levesque et al., 2005), and have numerous clinical applications (Payne, 1991).

During the course of daily handling of objects and exploratory touching, the fingerpad can experience considerable global deformation. For instance, $0.75 \mathrm{~N}$ of force could cause about $1.5 \mathrm{~mm}$ of normal deformation of a fingertip tapping at $0.5 \mathrm{~Hz}$ (Serina et al., 1997); $1.9 \mathrm{~N}$ of shearing force can induce about $4 \mathrm{~mm}$ of tangential deformation (Nakazawa et al., 2000). The global viscoelastic behavior of the fingerpad, thought to be important in the manipulative tasks, was the subject of several studies (Serina et al., 1997, 1998; Pawluk and Howe, 1999; Wu et al., 2003a,b; Jindrich et al., 2003; Pataky et al., 2005), but locally, the fingerpad skin also experiences considerable deformation: the act of sliding the fingertip on a flat glass surface can result in local tangential deformation of $\pm 30 \%$ (Levesque and Hayward, 2003). The viscoelasticity of human hairy skin has been demonstrated in vitro (Daly, 1982; Pan et al., 1997; Silver et al., 2001), but no study has yet examined viscoelastic properties of human fingerpad skin in vivo. 
We hypothesize that the fingerpad skin exhibits marked anisotropy, and substantial hysteretic losses under local cyclical loading, in addition to stiffening and time-dependent effects, and that these effects are significant under the large local deformations resulting from lateral traction loads. We developed an apparatus to specifically test the skin in vivo under these conditions. To evaluate elasticity, we stretched and sheared the skin with slow loading conditions. To evaluate anisotropy, we assumed the skin to be a homogeneous half-space and used this assumption to evaluate the "effective" Young's and shear moduli in specific directions, that is, the moduli of a homogenous material that would produce an equivalent behavior for a given test. It was then possible to quantitatively evaluate anisotropy as a function of ridge orientation. For viscoelasticity, we tested the skin for small deformations and identified a five-parameter model for relaxation and another for creeping. Finally, we evaluated the hysteretic loss of the skin under local cyclical loading. The skin was tested at a length scale of $0.3 \mathrm{~mm}$. Saint Venant's principle allowed us to ignore deformations at distances greater than one millimeter from the region of traction. As a result, we could characterize the dermis and epidermis behaviors where most of the low-threshold receptors are located (Paré et al., 2002, 2003; Nolano et al., 2003), and minimize the influence of the global deformation of the subcutaneous tissues.

\section{Methods and Materials}

\subsection{Subjects}

Twelve right-handed subjects, nine males and three females, volunteered to participate (Table 1). Only the right index finger skin was tested. No subjects reported having a history of skin pathology. The informed consent of the subjects was obtained in accordance with the requirements of the McGill University Policy on the Ethical Conduct of Research Involving Human Subjects.

Table 1

Subjects information (mean \pm standard deviation).

\begin{tabular}{lccccc}
\hline Protocol & \multicolumn{2}{c}{ Subjects } & Age & Body mass & Height \\
& ㅇ & o $^{\prime}$ & $($ years $)$ & $(\mathrm{kg})$ & $(\mathrm{cm})$ \\
\hline Tuning & 1 & 1 & $31 \pm 2$ & $65 \pm 14$ & $167 \pm 5$ \\
Elasticity & 3 & 5 & $28 \pm 3$ & $68 \pm 18$ & $174 \pm 15$ \\
Relaxation & 3 & 5 & $29 \pm 3$ & $67 \pm 18$ & $174 \pm 15$ \\
Creep & 2 & 6 & $29 \pm 3$ & $70 \pm 16$ & $176 \pm 12$ \\
Hysteresis & 1 & 2 & $31 \pm 2$ & $58 \pm 14$ & $168 \pm 3.0$ \\
\hline
\end{tabular}

Refer to Section 2.3 for a description of the protocols. 


\subsection{Apparatus}

We designed an apparatus that could deform the skin in the large deformation range (i.e. $\sim 100 \%$ ) and had wide operational bandwidth. With proper feedback control, it could load the skin under isotonic or under isometric conditions. Previously, we found that piezoelectric bimorph benders could stretch the skin to provide acceptable levels of sensation (Pasquero and Hayward, 2003). However, this was limited because cantilevers are too compliant. A cantilever parallel bender (Figure 1a) has this constituent equation (Smits et al., 1991):

$$
\delta=g f^{z}+\delta_{\text {Free }} V / V_{\max }
$$

where $\delta$ is the tip deflection, $g$ the compliance, $f^{z}$ the force at the tip, $\delta_{\text {Free }}$ the free deflection, $V$ the applied voltage, and $V_{\max }$ the maximum voltage, with

$$
g=\frac{l^{3}}{2 E w h^{3}} \quad \text { and } \quad \delta_{\text {Free }}=\frac{3 d_{31} l^{2}}{4 h^{2}} V_{\max }
$$

where $E$ is the piezo material's Young's modulus, $l$ and $w$ are the length and width of the layers, and $d_{31}$ is the piezoelectric coefficient. The compliance varies with $l^{3}$ and the free deflection with $l^{2}$. Hence, there exists an optimal length $l_{\text {opt }}$ that maximizes deflection for a given load. With the best commercial benders, we found that a cantilever could not deform the skin in the large range, even at $l_{\text {opt }}$. Now, if the benders are hinged at two places, termed "dual-pinned lever", as shown in Figure 1b, the compliance and the free deflection become (Supplementary Information):

$$
g=\frac{\left(l_{1}+l_{2}\right) l_{2}^{2}}{2 E w h^{3}} \quad \text { and } \quad \delta_{\text {Free }}=\frac{3 d_{31}\left(l_{1}+l_{2}\right) l_{2}}{4 h^{2}} V_{\max }
$$

To fabricate a cantilevered bender to achieve the same deflection as a dual-pinned bender, its

length $l$ would have to be $\sqrt{\left(l_{1}+l_{2}\right) l_{2}}$. Its compliance, $\left[\left(l_{1}+l_{2}\right) l_{2}\right]^{3 / 2} /\left(2 E w h^{3}\right)$, is always larger than that of the dual-pinned beam which is $\left[\left(l_{1}+l_{2}\right) l_{2}{ }^{2}\right] /\left(2 E w h^{3}\right)$. Moreover, a dual-pinned bender will always produce a higher blocked force for an equivalent deflection. By varying the ratio $l_{1} / l_{2}$ it is also possible to trade stiffness for free deflection.
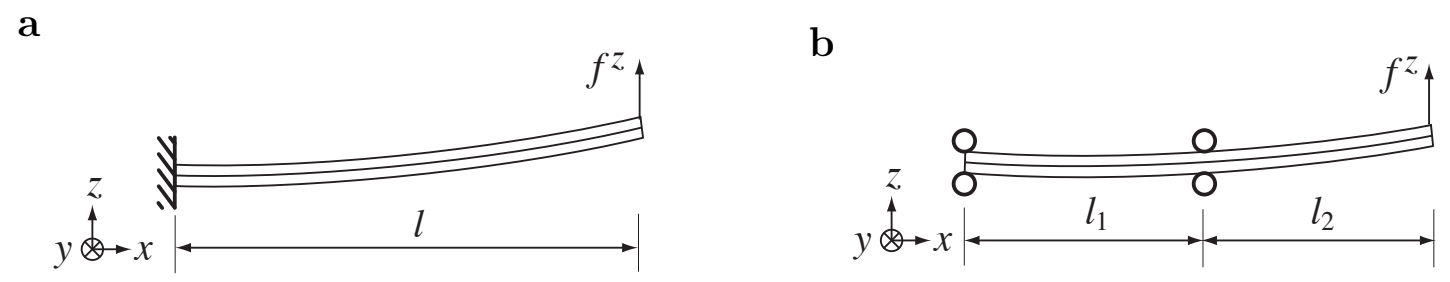

Fig. 1. a) Cantilered bender. b) Dual-pinned bender.

This was applied to the construction of the apparatus depicted in Figure 2. Two Y-poled parallel bimorph benders (Model T220-H4-303Y; Piezo Systems Inc., Cambridge, MA, USA) formed dual-pinned "tweezer". The upper pin was attached to a positioning stage (Model 4076m; Parker Hannifin Corporation, Rohnert Park, CA, USA) to vary the ratio $l_{1} / l_{2}$. A two-channel high voltage amplifier (Model 3584JM; Texas Instruments Inc., Dallas, TX, USA) drove the actuators. The tips 
of the benders were insulated with several layers of acrylic enamel. The deflection was found by measuring the displacement of a laser beam (Model 1107/P; JDS Uniphase, San Jose, CA, USA) reflected by a mirror glued near the tip (NT32-354; Edmund Industrial Optics, Barrington, NJ, USA). Displacement was detected by a lateral position sensing device (PSD, Model DL-10; UDT Sensors, Inc., Hawthorne, CA, USA). A vertically adjustable gutter and athletic tape immobilized the subject's finger.

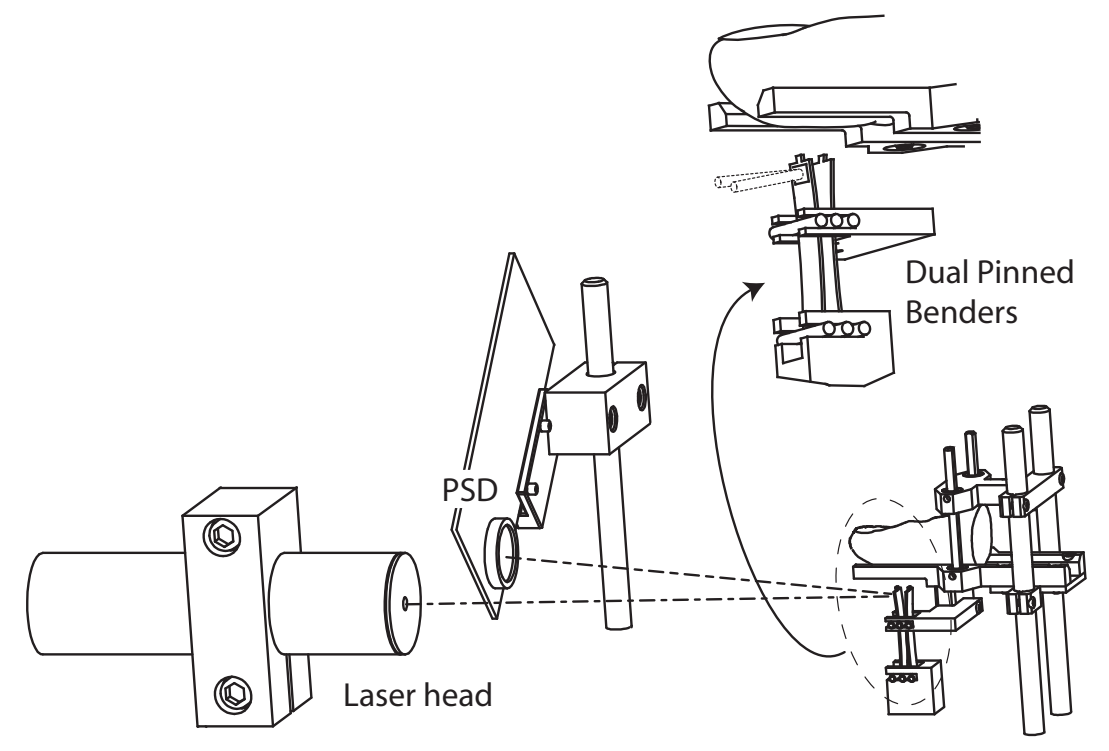

Fig. 2. Apparatus. The subject's finger was constrained in a vertically adjustable gutter that could be lowered until it touched the tips of a pair of dual-pinned piezoelectric benders. Micrometric stages made it possible to reach various places and orientations on the finger tip. Bender deflection was measured with a laser beam reflected by a small mirror attached near the tip of a bender and shinning onto a PSD detector. The piezoelectric actuators could be activated in closed loop to achieve isometric or isotonic loading.

The coefficient $d_{31}$ was calibrated by applying a quasi-static ramping voltage signal while recording the deflection. From the slope, the coefficient was estimated (Supplementary Information). By applying a known load to the tip of the bender (15 g weight), the Young's modulus was obtained. To minimize errors, the experiments were conducted for $l_{2}$ equal to $7,9,11,13$, and $15 \mathrm{~mm}$. The results agreed to within 5\%. The errors caused by actuator hysteresis were compensated by recording the unloaded voltage-deflection relationships.

When loaded, the deflection was a solution of $\delta=c f^{z}(\delta, t)+\delta_{\text {Free }} V / V_{\max }$, where $f^{z}(\delta, t)$ is a nonlinear time-varying function. The higher the $l_{2} / l_{1}$, the more compliant the structure and the larger the free deflection. In order to find a tradeoff, with the help of two subjects (Table 1), $l_{2}$ was varied by $2 \mathrm{~mm}$ steps, and the corresponding loaded deflections were recorded. This was repeated four times with each subject. The skin was allowed to recover from viscoelastic effects for at least 30 seconds between trials. Typical force-strain curves are seen in Figure 3. When $l_{2}=11 \mathrm{~mm}(l=30 \mathrm{~mm})$, the strain was maximized for both subjects. Analysis of variance (ANOVA) was performed on the maximal strains that the apparatus could create. The $p$-values were smaller than 0.001 when comparing deflections for $l_{2}=11 \mathrm{~mm}$ with those obtained for any 
other value.

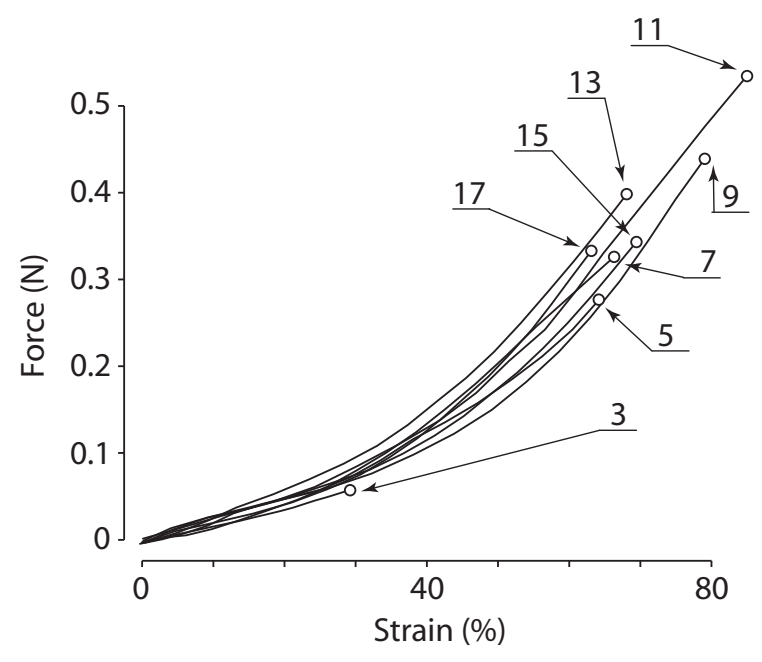

Fig. 3. Typical force-strain curves for different $l_{2}$ values. This data is for one subject. Deformation is optimized for $l_{2}=11 \mathrm{~mm}$ where the skin is tangentially strained at almost $100 \%$.

The apparatus created isometric or isotonic testing conditions by using a principle similar to the feedback modes in atomic force microscopy (Binnig et al., 1986). After calibration, the deflection $\delta$ can be written:

$$
\delta=k_{1} F+k_{2} V .
$$

For isotonic tests, the force $F=\left(\delta-k_{2} V\right) / k_{1}$ was regulated by a lead dynamic compensator $C_{t}$, Figure 4a. This provided the desired amount of bending regardless of the deflection caused by a load. For isometric tests, the deflection $\delta$ was regulated using another lead compensator $C_{m}$, Figure $4 \mathrm{~b}$, where the unknown force $F$ supplied by the load is a low frequency exogenous disturbance.

a

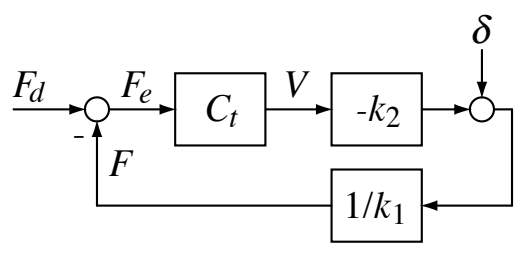

b

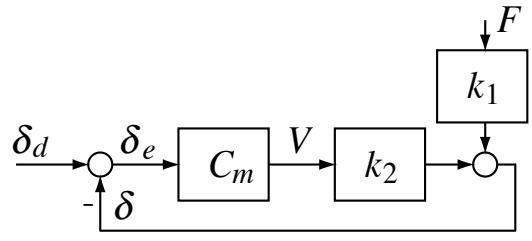

Fig. 4. a) Isotonic feedback. This configuration regulates the force applied by bending the actuators by the amount required to achieve a desired force. b) Isometric feedback. Here, the tip position is regulated regardless of the load.

\subsection{Procedure}

Subjects washed their hands and dried them with facial tissue before each test. A submillimetric area of the skin close to the papillary whorl was randomly selected. For all protocols, the tests were oriented along and across ridges. After each experiment, the tips of the benders were cleaned. Bonding was achieved by applying a thin layer of cyanoacrylate adhesive to the 
tips just before lowering the finger with a micrometric stage to make contact. The height of the fingerpad was adjusted so the skin did not appear to be pulled nor indented when examined using a 10x magnifying glass. Some tips were prepared for stretch tests and some others for shear tests. Traction surfaces are shown in Figures $5 \mathrm{a}$ and $5 \mathrm{~b}$.

a

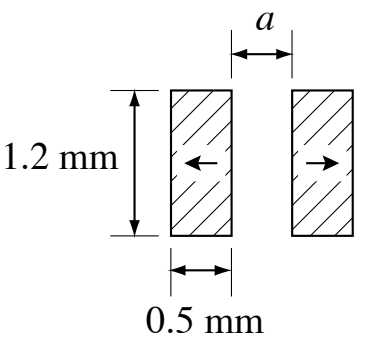

b

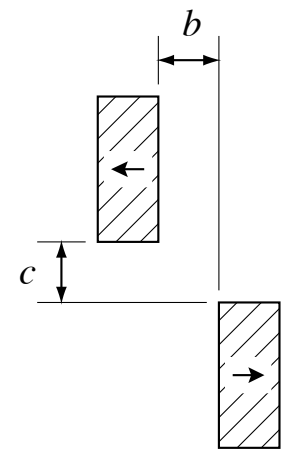

Fig. 5. Traction areas. The initial distances $a$ and $b$ were determined by the initial voltage applied to the actuators for a given test. a) Stretch tests. b) Shear tests.

Skin elasticity in tangential traction. Elasticity was examined both by stretching and shearing the skin. For stretching, the initial distance $a$ between the traction surfaces was $0.25 \mathrm{~mm}$. For shearing, $b$ was $0.25 \mathrm{~mm}$ and $c$ was $1.0 \mathrm{~mm}$.

Relaxation testing. Patches of skin $(a=0.4 \mathrm{~mm})$ were subjected to a pseudo-random lateral displacement signal switching from $0.015 \mathrm{~mm}$ to $0.06 \mathrm{~mm}$ applied tangentially at an average frequency of $0.2 \mathrm{~Hz}$ under isometric conditions. The skin behaved linearly in this range.

Creep testing. Patches of skin $(a=0.3 \mathrm{~mm})$ were stretch-loaded isotonically using a pseudorandom force signal. The signal had frequencies centered around $0.8 \mathrm{~Hz}$ and its amplitudes switched from $0.025 \mathrm{~N}$ to $0.25 \mathrm{~N}$. The skin behaved linearly in this range.

Hysteresis testing. Patches of skin $(a=0.3 \mathrm{~mm})$ were stretched cyclically. The driving voltages were ramped at a constant rate from $-80 \mathrm{~V}$ to $+80 \mathrm{~V}$, and back to $-80 \mathrm{~V}$. Unloading began immediately after loading and four different rates were used, yielding cycles of 10, 20, 40, and 80 seconds.

\subsection{Data processing}

All data were analyzed using Matlab ${ }^{\text {TM }}$ (The Math Works Inc., Natick, MA, USA) along with its system identification and statistics toolboxes.

Effective Young's and Shear modulus. For a homogenous isotropic half-space, the deformation displacement function of Boussinesq and Cerruti caused by a distributed tangential force along 
the $x$ axis is (Johnson, 1985):

$$
u_{x}=\frac{1}{4 \pi G} \iint_{S} q_{x}(\xi, \eta)\left(\frac{1}{\rho}+\frac{1-2 v}{\rho+z}+\frac{(\xi-x)^{2}}{\rho^{3}}-\frac{(1-2 v)(\xi-x)^{2}}{\rho(\rho-z)^{2}}\right) d \xi d \eta
$$

where $u_{x}$ is the displacement, $\rho=\sqrt{(\xi-x)^{2}+(\eta-y)^{2}+z^{2}}, S$ is the loading area, $q_{x}(\xi, \eta)$ is the distribution of the tangential force, $v$ and $G$ are the Poisson's ratio and shear modulus of the skin respectively. The Poisson ratio for soft tissues can be taken to be 0.5 . In addition, the tangential force distribution in the bonded area could be assumed to be uniform. The above expression simplifies to

$$
u_{x}=\frac{f_{T}}{4 \pi G A} \iint_{S}\left(\frac{1}{\rho}+\frac{(\xi-x)^{2}}{\rho^{3}}\right) d \xi d \eta=\frac{f_{T}}{4 \pi G A} \Psi(x),
$$

where $f_{T}$ is the tangential load and $A$ the traction area. The value of $\Psi(x)$ was evaluated for the traction areas in Figure 5 and the effective value of $E=2(1+\nu) G=3 G$ obtained from both stretch and shearing tests.

Relaxation and Creep Functions. Following Radok's suggestion as in (Johnson, 1985), the displacement along $x$ axis is:

$$
u_{x}(t)=\int_{0}^{t} c(t-\tau) \frac{d \frac{f_{T}(\tau)}{4 \pi G A} \Psi(x)}{d \tau} d \tau=\frac{\Psi(x)}{4 \pi G A} \int_{0}^{t} c(t-\tau) \frac{d f_{T}(\tau)}{d \tau} d \tau
$$

Since the system is causal, applying the Laplace transform yields

$$
\begin{aligned}
L\left[u_{x}(t)\right]=U_{x}(s) & =\frac{\Psi(x)}{4 \pi G A} \int_{0}^{\infty}\left[\int_{0}^{\infty} c(t-\tau) \frac{d F_{T}(\tau)}{d \tau} d \tau\right] e^{-s t} d t \\
& =\frac{\Psi(x)}{4 \pi G A} \int_{0}^{\infty} \frac{d F_{T}(\tau)}{d \tau} e^{-s \tau} d \tau \int_{0}^{\infty} c(t) e^{-s t} d t \\
& =\frac{\Psi(x)}{4 \pi G A} s F_{T}(s) C(s)
\end{aligned}
$$

Modeling the response as that of a viscoelastic solid represented by a model as in Figure 6, the transfer function from input displacement $u$ to output force $f_{T}$ is found to be:

$$
f_{T}=\mu_{0} u+\mu_{1} u_{1}+\mu_{2} u_{2}, \quad f_{T}=\mu_{0} u+\eta_{1} \dot{u}_{1}+\eta_{2} \dot{u}_{2} .
$$

After calculations,

$$
\frac{F_{T}(s)}{u(s)}=R(s)=\frac{1}{C(s)}=\mu_{0}+\frac{\eta_{1} \mu_{1} s}{\mu_{1}+\eta_{1} s}+\frac{\eta_{2} \mu_{2} s}{\mu_{2}+\eta_{2} s}
$$

For a more compact form, let $E_{R}=\mu_{0}, \tau_{1}=\frac{\eta_{1}}{\mu_{1}}, \sigma_{1}=\frac{\eta_{1}}{\mu_{0}}\left(1+\frac{\mu_{0}}{\mu_{1}}\right), \tau_{2}=\frac{\eta_{2}}{\mu_{2}}$, and $\sigma_{2}=\frac{\eta_{2}}{\mu_{0}}\left(1+\frac{\mu_{0}}{\mu_{2}}\right)$. The relaxation function $R(s)$ and the creep function $C(s)$ then are:

$$
R(s)=\frac{1}{C(s)}=\frac{E_{R}+\left(\sigma_{1}+\sigma_{2}\right) s+\left(\sigma_{1} \tau_{2}+\sigma_{2} \tau_{1}-E_{R} \tau_{1} \tau_{2}\right) s^{2}}{\left(1+\tau_{1} s\right)\left(1+\tau_{2} s\right)} .
$$


Using the bilinear transformation $s=\frac{2}{T}(z-1) /(z+1)$ to substitue $s$, we obtained a discrete-time domain transfer function that could be identified using an ARMAX procedure.

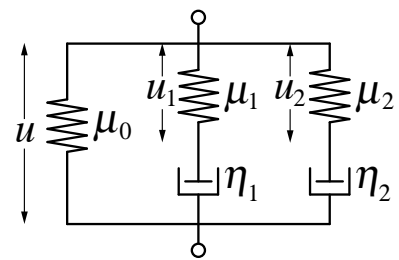

Fig. 6. Second order linear viscoelastic material model. This model yielded a much better fit than a first order viscoelastic model due to the presence of two relaxation rates.

\section{Results}

\subsection{Young's modulus}

A typical nonlinear skin force-strain curve is seen in the Figure 7. Because of the tangential traction, there was no clearly defined "knee" in the curve although the skin became very stiff beyond $50 \%$ strain. The means and standard deviations of the Young's modulus are collected in Figure 8 for all eight subjects. The moduli found by stretching the skin along ridges were consistently higher than those found by stretching it across ridges. In Figure 8 the results are sorted by order of decreasing differences between directions. For each subject, a two-sample, twotailed Student $t$-tests was conducted on the Young's moduli found by stretching and shearing along and across ridges which all rejected the null hypothesis (5\% significance level).

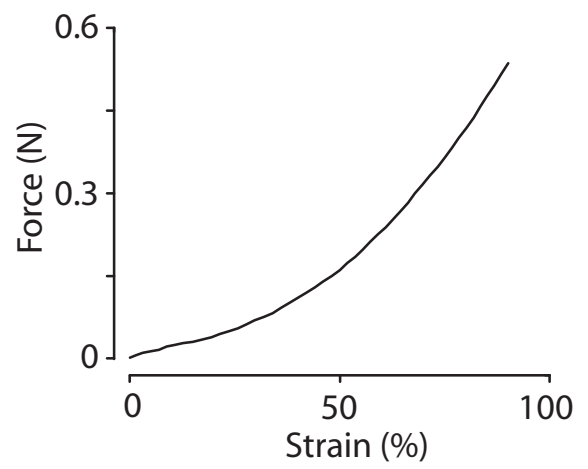

Fig. 7. Nonlinear force-strain relationship of the skin of one subject.

ANOvA was used to compare the mean Young's modulus found by stretching/shearing the skin along ridges with that across ridges, see Table 2. The Young's moduli were significantly dependent on the direction of the deformation relative to the orientation of the ridges $(p=0.012$ and $p=0.023$ for the stretching and the shearing conditions respectively). In both conditions, the skin was stiffer along the ridges than across them. Conversely, ANOVA tests yielded no significant difference between two groups of Young's moduli measured along proximal-distal direction versus 


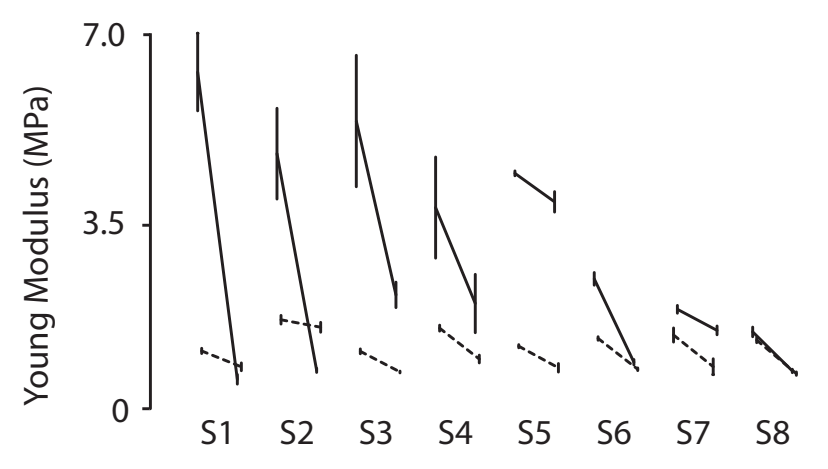

Fig. 8. Skin moduli of eight subjects listed on the horizontal axis. Solid line shows the effective Young's moduli measured by stretching the skin, and the dashed line by shearing the skin. For each subject, left: effective Young's moduli of the skin along the ridges; right: across the ridges. For some subjects, the moduli found by stretching traction is highly dependent on the ridge orientation but less for others (solid lines). The moduli found by shearing are less sensitive to ridge orientation, yet consistently different (dashed lines).

the radial-ulnar direction ( $p=0.474$ for the stretching condition, and $p=0.675$ for the shearing condition), implying that the stiffness difference was related to the direction of ridge, but not to the orientation of the test with respect to the finger.

Table 2

Mean \pm standard deviation skin moduli for all eight subjects.

\begin{tabular}{lcc}
\hline & $\begin{array}{c}\text { Along ridges } \\
(\mathrm{MPa})\end{array}$ & $\begin{array}{c}\text { Across ridges } \\
(\mathrm{MPa})\end{array}$ \\
\hline Stretching & $3.61 \pm 1.73$ & $1.54 \pm 1.08$ \\
Shearing & $1.36 \pm 0.27$ & $0.96 \pm 0.34$ \\
\hline
\end{tabular}

\subsection{Relaxation}

Figure 9 shows typical relaxation when the skin is subjected to a pseudo-random isometric test. The uniaxial force decreased rapidly over the first 5-8 seconds, then the relaxation rate was low until the force reached a steady value. This justified the choice of a model having one elastic term and two time constants. The five parameters of the linear standard solid were identified for each subject and listed in Table 3. ANOva tests found no significant differences along and across ridges. 

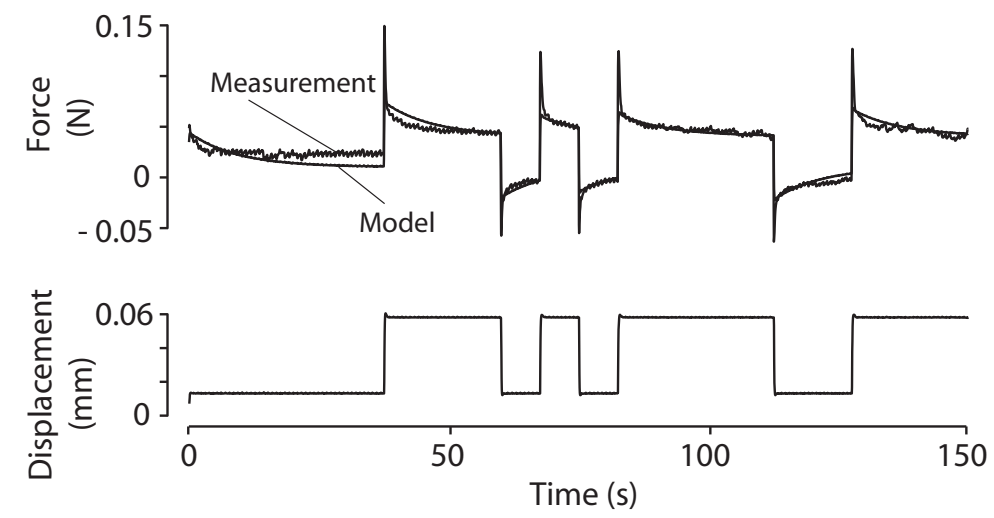

Fig. 9. Relaxation behavior of the skin. For many subjects, after each displacement transient, the force dropped abruptly and then slowly relaxed over a long time period.

Table 3

Relaxation protocol parameter fit for all eight subjects.

\begin{tabular}{|c|c|c|c|c|c|c|c|c|c|c|c|c|}
\hline & \multicolumn{6}{|c|}{ Along } & \multicolumn{6}{|c|}{ Across } \\
\hline & $\mu_{0}$ & $\mu_{1}$ & $\eta_{1}$ & $\mu_{2}$ & $\eta_{2}$ & $r^{2}$ fit & $\mu_{0}$ & $\mu_{1}$ & $\eta_{1}$ & $\mu_{2}$ & $\eta_{2}$ & $r^{2}$ fit \\
\hline S1 & 0.69 & 0.44 & 0.18 & 0.63 & 0.001 & $81 \%$ & 0.93 & 0.85 & 0.06 & 1.95 & 0.002 & $78 \%$ \\
\hline $\mathrm{S} 2$ & 0.54 & 0.62 & 0.24 & 5.18 & 0.053 & $81 \%$ & 0.35 & 0.18 & 0.36 & 0.37 & 0.001 & $82 \%$ \\
\hline S3 & 1.26 & 0.70 & 0.52 & 8.55 & 0.044 & $55 \%$ & 0.85 & 1.48 & 0.89 & 9.76 & 0.113 & $50 \%$ \\
\hline $\mathrm{S} 4$ & 0.87 & 0.91 & 1.12 & 17.79 & 0.095 & $64 \%$ & 0.49 & 0.88 & 1.43 & 13.36 & 0.089 & $76 \%$ \\
\hline S5 & 2.97 & 0.56 & 1.85 & 0.88 & 0.022 & $82 \%$ & 0.93 & 0.90 & 0.40 & 2.52 & 0.124 & $86 \%$ \\
\hline S6 & 0.92 & 1.26 & 0.83 & 4.92 & 0.169 & $78 \%$ & 1.01 & 1.73 & 1.16 & 4.07 & 0.145 & $79 \%$ \\
\hline S7 & 0.43 & 1.07 & 0.40 & 10.81 & 0.084 & $88 \%$ & 0.60 & 0.32 & 0.50 & 6.13 & 0.085 & $93 \%$ \\
\hline S8 & 0.72 & 0.40 & 0.31 & 9.38 & 0.064 & $83 \%$ & 0.99 & 0.30 & 1.60 & 8.09 & 0.051 & $86 \%$ \\
\hline
\end{tabular}

All eight subjects were tested. Much variability across subjects was observed.

\subsection{Creep}

Figure 10 shows a typical creep curve when the skin is subjected to a pseudo-random isotonic test. When stretched by a constant force, the skin elongated at high rate. The deformation decreased slower than it increased, implying that hysteresis existed. A fit for the five parameters of the linear standard solid was attempted for each subject. These are listed in Table 4. AnovA failed to find significant differences among tests along and across ridges. 


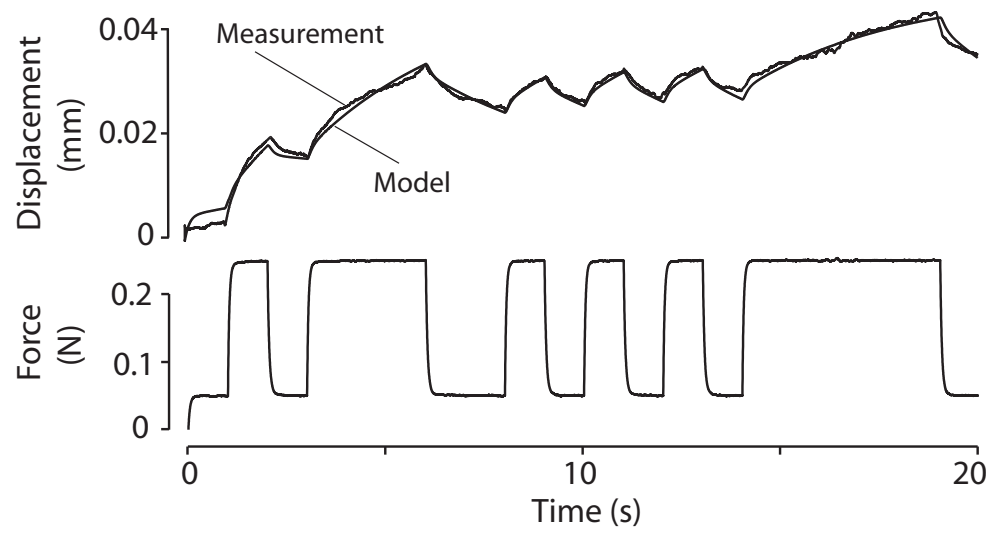

Fig. 10. Creeping behavior of the skin. Force transients caused the skin to creep about twice slower than it relaxed. Visual inspection and comparison can be misleading.

Table 4

Creep protocol parameter fit for all eight subjects.

\begin{tabular}{|c|c|c|c|c|c|c|c|c|c|c|c|c|}
\hline & \multicolumn{6}{|c|}{ Along } & \multicolumn{6}{|c|}{ Across } \\
\hline & $\mu_{0}$ & $\mu_{1}$ & $\eta_{1}$ & $\mu_{2}$ & $\eta_{2}$ & $r^{2}$ fit & $\mu_{0}$ & $\mu_{1}$ & $\eta_{1}$ & $\mu_{2}$ & $\eta_{2}$ & $r^{2}$ fit \\
\hline S1 & 2.20 & 7.92 & 1.10 & 15.09 & 0.40 & $93 \%$ & 0.41 & 4.99 & 1.67 & 11.34 & 0.51 & $90 \%$ \\
\hline S2 & 1.62 & 1.37 & 2.38 & 37.65 & 1.86 & $83 \%$ & 0.17 & 1.82 & 3.92 & 42.65 & 1.19 & $81 \%$ \\
\hline S3 & 0.71 & 7.15 & 2.46 & 10.84 & 0.18 & $89 \%$ & 0.75 & 2.88 & 3.68 & 43.53 & 2.76 & $79 \%$ \\
\hline $\mathrm{S} 4$ & 0.04 & 1.69 & 16.44 & 19.83 & 2.60 & $78 \%$ & 0.23 & 1.31 & 32.04 & 13.76 & 2.83 & $86 \%$ \\
\hline S5 & 0.14 & 0.48 & 1.28 & 2.75 & 0.48 & $76 \%$ & 0.34 & 4.15 & 54.96 & 6.59 & 2.92 & $76 \%$ \\
\hline S6 & 0.11 & 0.48 & 1.19 & 1.40 & 0.55 & $93 \%$ & 0.20 & 0.89 & 1.63 & 1.44 & 0.25 & $91 \%$ \\
\hline S7 & 1.42 & 6.38 & 2.86 & 15.14 & 0.33 & $84 \%$ & 0.06 & 2.54 & 18.84 & 31.18 & 2.54 & $80 \%$ \\
\hline S8 & 1.09 & 4.13 & 4.74 & 33.86 & 1.18 & $86 \%$ & 0.99 & 6.55 & 2.23 & 19.33 & 1.13 & $85 \%$ \\
\hline
\end{tabular}

All eight subjects were tested. Much variability across subjects was observed.

\subsection{Hysteresis}

The skin of all three subjects exhibited strong hysteresis with substantial energy loss $(81.1 \%$ on average). Typical hystereses for one subject are shown in Figure 11 for various loading and unloading times. The responses are highly repeatable and essentially invariant for times longer than 20 seconds. It is only with faster stimulation (5 second loading and 5 second unloading) that the losses tended to diminish. ANOVA found no significant effects of the ridge orientation $(p=0.672)$, and no significant effect of the loading and unloading time, once large enough $(p=0.826)$. 


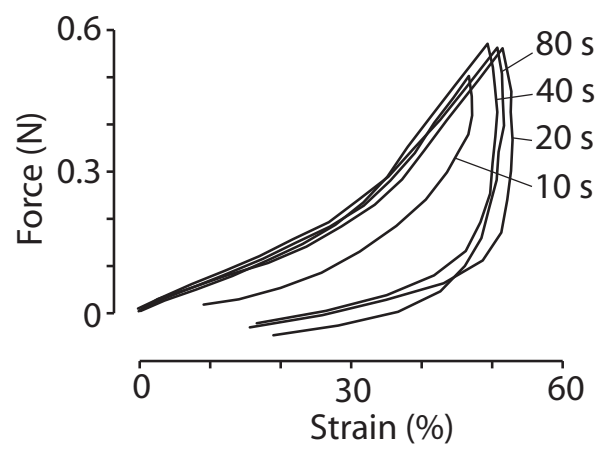

Fig. 11. Hystereses of the skin of one subject. The hysteretic behavior is by and large invariant from the cycle period until it becomes short enough (10 s). The loss then decrease.

\section{Discussion}

Incremental loading, both stretching and shearing, induced nonlinear behaviors, including stiffening at high strains. Pan et al. (1997) found the average Young's modulus of human forearm skin in vivo, strained at $40 \%$, to be $458.7 \mathrm{kPa}$, and Silver et al. (2001) found the slope at high strain $(\geq 40 \%)$ of the stress-strain curve of the human abdomen skin in vitro to be $18.8 \mathrm{MPa}$. The discrepancy with our results (mean effective Young's modulus is 3.61 Mpa along the ridges, and 1.54 Mpa across the ridges) can be explained by the fact that the stratum corneum layer of the glabrous skin is at least 4 times thicker than that of hairy skin (Cauna, 1954); therefore, the glabrous skin in the small deformation range appears to be much stiffer than the hairy skin. Also, our study was performed in vivo.

There was much variance in the effective Young's moduli among the subjects. The stiffest skin (along the ridges) was about 10 times stiffer than the softest skin (across the ridges). This might be attributed to the different thickness of the stratum corneum layer from subject to subject. This is consistent with previous findings which indicated that there is great variance in both the absolute psychophysical threshold in terms of mechanical stimulus amplitude (Lindblom and Lindstrom, 1976; Vallbo and Johansson, 1976; Johansson and Vallbo, 1979a, 1983) and neural thresholds (Knibestol, 1973, 1975; Johansson and Vallbo, 1979a, 1983).

Previous works addressed the directional effects of skin. Some of these effects are strongly correlated with the Langer's lines (Ridge and Wright, 1966; Gibson et al., 1969; Stark, 1977). Some others are related to the body geometry. Daly (1982) reported that a skin specimen taken from the abdomen along to the cranio-caudal axis could be stretched longer than one taken perpendicularly. Our results imply that the directional effect of the fingerpad skin is not related to the finger geometry but to the orientation of the papillary ridges. This might be due to the fact that the skin micro-geometry may generate unevenly distributed stress fields across the ridge grooves (Maeno et al., 1998; Gerling and Thomas, 2005), eventually contributing to the directionality of the responses of receptors such as the Merkel discs and Meissner corpuscles, which are structurally associated to the papillary ridges (Vallbo and Johansson, 1978). The directionality of mechanoreceptive afferents innervating in the glabrous skin has been observed 
for long time. The response of individual afferents were found to be dependent on the direction of skin stretch (Johansson and Vallbo, 1983) or on the direction of stimulus movement (LaMotte and Srinivasan, 1987b,a). The populations of each class of three classes of cutaneous afferents (slowly adapting I, slowly adapting II, and rapidly adapting I) were biased to a certain direction; this is perhaps because of the asymmetric strains caused by the ridge orientations (Birznieks et al., 2001).

It is widely held that Langer's lines are associated with the orientation of collagen fibres in the dermis. Our literature review did not reveal any findings related to the orientation of Langer's lines in human fingerpad skin (Gibson et al., 1969). It is conceivable that the papillary ridges might be aligned with the Langer's lines if they exist in the fingerpad. Our data showed that the directional stiffness variance measured by stretching the skin was significantly larger than when shearing the skin, implying that stiffness directionality occurs locally. This might explain the typical fore-aft finger movements used by people when judging the texture or roughness of an unknown surface in a possible attempt to find trajectories that maximize strain.

While the skin viscoelasticity has been addressed previously (Pereira et al., 1991; Silver et al., 2001), this study is the first to investigate relaxation and creep in the living glabrous skin. Many studies used exponential fit methods to quantify the parameters of viscoelasticity (Jamison, 1968; Pataky et al., 2005). Although these methods can evaluate precisely the discrete spectrum associated with characteristic frequencies (Fung, 1993), the ARMAX approach we used in this study identified the continuous spectrum of the relaxation function and creep function of the skin, which would be used for the development of dynamic finite elements models.

Our data showed that the relaxation time of skin under tangential traction is around 10 seconds. This result agrees well with the results of Pataky et al. (2005), who showed that the mean relaxation time of fingerpads subjected to tangential loads was about 11.2 seconds, and that of Moy et al. (2000a) and Pawluk and Howe (1999), which indicated that the relaxation time of fingerpads subjected to normal loads was around 8 seconds. Since the relaxation has been observed to strongly bias the tactile perception (Moy et al., 2000a), it is plausible that the viscoelastic behavior of the skin might contribute to the neuronal dynamics such as adaptation. The creep time of the skin was found in this study to be about twice that of relaxation, implying that the viscous migration of fluids in the skin (Jamison, 1968; Pan et al., 1998), behaves differently when the flow is in or out the loaded skin. Similarly, these differences between loading conditions might affect the response of the receptors.

Fearing and Hollerbach (1984) found that, theoretically, the optimum ridge spacing should be about $3 \mathrm{~mm}$ for sensing strain when considering the finger to be a homogeneous elastic medium. The viscoelastic properties of actual skin might modify the time course of strain propagation in a manner that necessitates much denser ridges to sense strain optimally.

There was a surprisingly large amount of hystereric loss for localized deformation. This is most likely attributable to the changing special arrangement of the skin collagen fiber network: the fibrils of the skin collagen fiber network are entangled when relaxed, then are gradually straightened and aligned to carry the load (Kenedi et al., 1965; Gibson et al., 1969; Daly, 1982). 
Once aligned, the fiber network might not recover its relaxed state before a long period of time and/or different loading conditions occur. It would be speculative to try to distinguish the relative contributions of viscoelasticity and a rate-independent internal structural modification, although both are likely to participate in the observed hysteresis.

This study described a method for in vivo testing the local biomechanical properties of soft tissue. The results could be useful to the set up of numerical models and for other types of investigations such as diagnosis of skin condition or the study of aging.

\section{Acknowldgedments}

The authors would like to thank Allan M. Smith, Christopher I. Moore, Garrett B. Stanley, and the anonymous reviewers for insightful comments. This research was supported IRIS, the Institute for Robotics and Intelligent Systems. Qi Wang would like to thank McGill University for an Eric L. Adler Fellowship and Vincent Hayward would like to thank NSERC the Natural Sciences and Engineering Research Council of Canada for a Discovery Grant.

\section{References}

Biggs, J., Srinivasan, M. A., 2002. Tangential versus normal displacements of skin: Relative effectiveness for producing tactile sensations. In: Proceedings of the 10th International Symposium on Haptic Interfaces for Virtual Environment and Teleoperator Systems. pp. 121-128.

Binnig, G., Quate, C. F., Gerber, C., 1986. Atomic force microscope. Physics Review Letters 56 (9), 930-933.

Birznieks, I., Jenmalm, P., Goodwin, A. W., Johansson, R. S., 2001. Encoding of direction of fingertip forces by human tactile afferents. Journal of Neuroscience 21, 8222-8237.

Bisley, J. W., Goodwin, A. W., Wheat, H. E., 2000. Slowly adapting type i afferents from the sides and end of the finger respond to stimuli on the center of the fingerpad. Journal of Neurophysiology 84, 57-64.

Cauna, N., 1954. Nature and functions of the papillary ridges of the digital skin. The Anatomical Record 119, 449-468.

Daly, C. H., 1982. Biomechanical properties of dermis. Journal of Investigative Dermatology 79 Supplement 1, 17-20.

Drewing, K., Fritschi, M., Zopf, R., Ernst, M., Buss, M., 2005. First evaluation of a novel tactile display exerting shear force via lateral displacement. ACM Transactions on Applied Perception $2(2), 118-131$.

Edin, B. B., Johansson, N., 1995. Skin strain patterns provide kinaesthetic information to the human central nervous system. Journal of Physiology 487, 243-251.

Fearing, R. S., Hollerbach, J. M., 1984. Basic solid mechanics for tactile sensing. In: Proc. IEEE International Conference on Robotics and Automation. pp. 266-275. 
Fung, Y. C., 1993. Biomechanics: Mechanical properties of living tissues. Springer-Verlag New York, Inc.

Gerling, G., Thomas, G., 2005. The effect of fingertip microstructures on tactile edge perception. In: First Joint Eurohaptics Conference and Symposium on Haptic Interfaces for Virtual Environment and Teleoperator Systems. pp. 63-72.

Gibson, T., Stark, H., Evans, J. H., 1969. Directional variation in extensibility of human skin in vivo. Journal of Biomechanics 2, 201-202.

Goodwin, A. W., Macefield, V. G., Bisley., J. W., 1997. Encoding object curvature by tactile afferents from human fingers. Journal of Neurophysiology 78, 2881-2888.

Goodwin, A. W., Wheat, H. E., 2004. Sensory signals in neural populations underlying tactile perception and manipulation. Annual Review of Neuroscience 27, 53-77.

Jamison, C. E., 1968. Viscoelastic properties of soft tissue by discrete model characterization. Journal of Biomechanics 1, 33-46.

Jindrich, D. L., Zhou, Y., Becker, T., Dennerlein, J. T., 2003. Non-linear viscoelastic models predict fingertip pulp force-displacement characteristics during voluntary tapping. Journal of Biomechanics 36, 497-503.

Johansson, E. S., Birznieks, I., 2004. First spikes in ensembles of human tactile afferents code complex spatial fingertip events. Nature Neuroscience 7(2), 170-177.

Johansson, R. S., Vallbo, A. B., 1979a. Detection of tactile stimuli. thresholds of afferent units related to psychophysical thresholds in the human hand. Journal of Physiology 197, 405-422.

Johansson, R. S., Vallbo, A. B., 1979b. Tactile sensitivity in the human hand: Relative and absolute densities of four types of mechanoreceptive units in glabrous skin. Journal of Physiology 286, 283-300.

Johansson, R. S., Vallbo, A. B., 1983. Tactile sensory coding in the glabrous skin of the human hand. Trends Neuroscience 6, 27-31.

Johansson, R. S., Westling, G., 1984. Roles of glabrous skin receptors and sensorimotor memory in automatic control of precision grip when lifting rougher or more slippery objects. Experimental Brain Research 56, 550-564.

Johansson, R. S., Westling, G., 1987. Signals in tactile afferents from the fingers eliciting adaptive motor responses during precision grip. Experimental Brain Research 66, 141-154.

Johnson, K. L., 1985. Contact mechanics. Cambridge University Press.

Johnson, K. O., 2001. The roles and functions of cutaneous mechanoreceptors. Current Opinion in Neurobiology 11, 455-461.

Johnson, K. O., Hsiao, S. S., 1992. Neural mechanisms of tactual form and texture perception. Annual Review of Neuroscience 15, 227-250.

Kenedi, R. M., Daly, C. H., Gibson, T., 1965. The determination, significance and application of the biomechanical characteristics of human skin. In: Digest of 6th International Conference of Medical Electronics and Biological Engineering.

Knibestol, M., 1973. Stimulus response functions of rapidly adapting mechanoreceptors in the human glabrous skin area. Journal of Physiology 232, 427-452.

Knibestol, M., 1975. Stimulus response functions of slowly adapting mechanoreceptors in the human glabrous skin area. Journal of Physiology 245, 63-80.

LaMotte, R. H., Srinivasan, M. A., 1987a. Tactile discrimination of shape: Responses of rapidly adapting mechanoreceptive afferents to a step stroked across the monkey fingerpad. Journal of 
Neuroscience 7, 1672-1681.

LaMotte, R. H., Srinivasan, M. A., 1987b. Tactile discrimination of shape: Responses of slowly adapting mechanoreceptive afferents to a step stroked across the monkey fingerpad. Journal of Neuroscience 7, 1655-1671.

Levesque, V., Hayward, V., 2003. Experimental evidence of lateral skin strain during tactile exploration. In: Proceedings of Eurohaptics 2003. pp. 261-275.

Levesque, V., Pasquero, J., Hayward, V., Legault, M., 2005. Display of virtual Braille dots by lateral skin deformation: Feasibility study. ACM Transactions on Applied Perception 2 (2), $132-149$.

Lindblom, U., Lindstrom, B., 1976. Tactile thresholds of normal and blind subjects on stimulation of finger pads with short mechanical pulses of variable amplitude. In: Zotterman, Y. (Ed.), Sensory Functions of the Skin in Primates, with special reference to Man. Pergamon Press Ltd., pp. 105-111.

Maeno, T., Kobay-Ashi, K., Yamazaki, N., 1998. Relationship between the structure of human finger tissue and the location of tactile receptors. JSME International Journal 41, 94-100.

Moy, G., Singh, U., Tan, E., Fearing, R., 2000a. Human psychophysics for teletaction system design. Haptics-e 1 (3).

Moy, G., Wagner, C., Fearing, R. S., 2000b. A compliant tactile display for teletaction. In: Proceedings of the IEEE International Conference on Robotics and Automation. pp. 34093415.

Nakazawa, N., Ikeura, R., H.Inooka, 2000. Characteristics of human fingertips in the shearing direction. Biological Cybernetics 82, 207-214.

Nolano, M., Provitera, V., Crisci, C., 2003. Quantification of myelinated endings and mechanoreceptors in human digital skin. Annals of Neurology 54, 197-205.

Pan, L., Zan, L., Foster, F. S., 1997. In vivo high frequency ultrasound assessment of skin elasticity. In: Proceedings of the IEEE Ultrasonics Symposium. pp. 1087-1091.

Pan, L., Zan, L., Foster, F. S., 1998. Ultrasonic and viscoelastic properties of skin under transverse mechanical stress in vitro. Ultrasound in Medicine \& Biology 24, 995-1007.

Paré, M., Behets, C., Cornu, O., 2003. Paucity of presumed ruffini corpuscles in the index fingerpad of humans. Journal of Comparative Neurology 356, 260-266.

Paré, M., Smith, A. M., Rice, F. L., 2002. Distribution and terminal arborizations of cutaneous mechanoreceptors in the glabrous finger pads of the monkey. Journal of Comparative Neurology 445, 347-359.

Pasquero, J., Hayward, V., 2003. stress: A practical tactile display system with one millimeter spatial resolution and $700 \mathrm{~Hz}$ refresh rate. In: Proceedings of Eurohaptics. pp. 94-110.

Pataky, T. C., Latash, M. L., Zatsiorsky, V. M., 2005. Viscoelastic response of the finger pad to incremental tangential displacements. Journal of Biomechanics 38, 1441-1449.

Pawluk, D. T. V., Howe, R., 1999. Dynamic contact of the human fingerpad against a flat surface. Journal of Biomechanical Engineering 121, 605-611.

Payne, P. A., 1991. Measurement of properties and function of skin. Clinical Physics and Physiological Measurements 12, 105-129.

Pereira, J. M., Mansour, J. M., Davis, B. R., 1991. Dynamic measurement of the viscoelastic properties of skin. Journal of Biomechanics 24, 157-16.

Phillips, J. R., Johnson, K. O., 1981a. Tactile spatial resolution: II. neural representation of bars, 
edges, and gratings in monkey primary afferents. Journal of Neurophysiology 46, 1192-1203.

Phillips, J. R., Johnson, K. O., 1981b. Tactile spatial resolution. III. a continuum mechanics model of skin predicting mechanoreceptor responses to bars, edges, and gratings. Journal of Neurophysiology 46, 1204-1225.

Ridge, M. D., Wright, V., 1966. The directional effects of skin: a bio-engineering study of skin particular reference to langer's lines. Journal of Investigative Dermatology 46, 341-346.

Serina, E. R., C. D. Mote, J., Rempel, D., 1997. Force response of the fingertip pulp to repeated compression - effects of loading rate, loading angle and anthropometry. Journal of Biomechanics 30, 1035-1040.

Serina, E. R., Mockensturm, E., Jr., C. D. M., Rempel, D., 1998. A structural model of the forced compression of the fingertip pulp. Journal of Biomechanics 31, 639-646.

Silver, F. H., Freeman, J. W., DeVore, D., 2001. Viscoelastic properties of human skin and processed dermis. Skin Research and Technology 7, 18-23.

Smits, J. G., Dalke, S. I., Cooney, T. K., 1991. The constituent equations of piezoelectric bimorphs. Sensors and Actuators A 28, 41-61.

Srinivasan, M. A., Dandekar, K., 1996. An investigation of the mechanics of tactile sense using two dimensional models of the primate fingertip. Journal of Biomechanical Engineering 118, $48-55$.

Srinivasan, M. A., LaMotte, R. H., 1987. Tactile discrimination of shape: Responses of slowly and rapidly adapting mechanoreceptive afferents to a step indented into the monkey fingerpad. Journal of Neuroscience 7, 1682-1697.

Stark, H. L., 1977. Directional variations in the extensibility of human skin. British Journal of Plastic Surgery 30, 105-114.

Vallbo, A. B., Johansson, R. S., 1976. Skin mechanoreceptors in the human hand: Neural and psychophysical thresholds. In: Zotterman, Y. (Ed.), Sensory Functions of the Skin in Primates, with special reference to Man. Pergamon Press Ltd., pp. 185-199.

Vallbo, A. B., Johansson, R. S., 1978. The tactile sensory innervation of the glabrous skin of the human hand. In: Gordon, G. (Ed.), Active Touch. pp. 29-54, oxford, U.K.: Pergamon Press.

Wu, J. Z., Dong, R., Schopper, A. W., Smutz, W. P., 2003a. Analysis of skin deformation profiles during sinusoidal vibration of fingerpad. Annals of Biomedical Engineering 31, 867-878.

Wu, J. Z., Dong, R. G., Smutz, W. P., Schopper, A. W., 2003b. Modeling of time-dependent force response of fingertip to dynamic loading. Journal of Biomechanics 36, 383-392. 